\title{
Coagulation status following therapeutic plasma exchange using citrate in kidney transplant recipients
}

\author{
Running head: Citrate effect after plasma exchange \\ Chisa Yamada ${ }^{1}$, Steven W. Pipe ${ }^{2}$, Lili Zhao ${ }^{3}$, Alan B. Leichtman ${ }^{4}$, Milagros Samaniego ${ }^{4}$, \\ Randall S. Sung ${ }^{5}$, Robertson D. Davenport ${ }^{1}$ \\ ${ }^{1}$ Department of Pathology \\ University of Michigan \\ ${ }^{2}$ Department of Pediatrics/Pathology \\ University of Michigan \\ ${ }^{3}$ Department of Biostatistics \\ University of Michigan \\ ${ }^{4}$ Department of Internal Medicine \\ University of Michigan \\ ${ }^{5}$ Department of Surgery \\ University of Michigan \\ Address for correspondence: \\ Chisa Yamada, M.D. \\ University of Michigan \\ Blood Bank / Transfusion Medicine \\ Department of Pathology \\ 1500 E. Medical Center Dr. \\ SPC 5054, 2F228 UH \\ Ann Arbor, MI 48109-5054 \\ Tel: (734) 936-6776 \\ Fax: (734) 936-6888 \\ E-mail: yamadac@med.umich.edu \\ Reprint will not be available from the author.
}

Source of support: University of Michigan Pathology Internal Grant supported lab tests and study coordinators' fees.

I should like to mention here that I and all of my co-authors have read the AABB's policy on Conflict of Interest and none of us have any Conflicts of Interest or financial interests related to the manuscript.

Word count: 3,471

This is the author manuscript accepted for publication and has undergone full peer review but has not been through the copyediting, typesetting, pagination and proofreading process, which may lead to differences between this version and the Version record. Please cite this article as doi:10.1111/ trf.13803. 


\section{ABSTRACT}

BACKGROUND: Therapeutic plasma exchange (TPE) is increasingly used for treatment of antibody mediated rejection (AMR) following solid organ transplants. There is concern that TPE may increase risk of bleeding, although data are limited. After TPE, clot-based coagulation tests may not accurately represent the levels of coagulation factors due to the effect of citrate. We investigated protein levels of fibrinogen using antigen detection methodology (FibAg) and correlated results with a clot-based fibrinogen activity test (Fib). STUDY DESIGN: Nine kidney transplant recipients who received TPE for AMR were investigated. Fib, FibAg, PT/INR, PTT, factor X chromogenic activity (CFX), and ionized calcium (iCa) were measured at Pre/Post-TPE, 1, 3, 6, 9, 24 and 48 hours after the first TPE. RESULTS: Mean Fib/FibAg ratio before TPE was 1.08; therefore, all Fib values were normalized (n) by dividing by 1.08. Overall, the mean $n$ Fib/FibAg ratio at Post-TPE was 0.89 and returned to close to 1.0 at 6 hours after the first TPE. Decreases in nFib, FibAg, CFX, and increases in PT/INR and PTT at Post-TPE were observed. The lowest Fib, FibAg, CFX, platelet and iCa levels were still at levels that would be considered sufficient for hemostasis at all-time points. CONCLUSION: The mean nFib/FibAg ratio after TPE was 0.89 and normalized in 6 hours, which demonstrates a persistent effect of citrate for up to 6 hours. Therefore, similar data observed in clot-based tests of PT/INR and PTT may be falsely elevated up to 6 hours after TPE due to the citrate effect.

Key Words: Therapeutic plasma exchange, hemostasis, solid organ transplants. 


\section{INTRODUCTION}

In recent years, therapeutic plasma exchange (TPE), combined with immunomodulation, has been increasingly used for treatment for antibody mediated rejection (AMR), especially in kidney transplant recipients. At our institution, many patients start TPE shortly after the histological diagnosis of AMR; and the occurrence of bleeding complications after kidney allograft biopsy in the period of 2010 - 2011 was 5 times higher in patients who received TPE than in patients who did not receive TPE; $1.03 \%$ in all patients vs. $5.17 \%$ in patients who received TPE within 5 days of the biopsy (unpublished data, courtesy of nephrology service at our institution). One of the possible causes of this can be coagulopathy after TPE procedures. The most commonly used coagulation tests, including PT/INR, PTT, coagulation factor assays and fibrinogen activity (Fib) are based on clotting time, and are influenced by the presence of citrate, which is used for TPE procedures. Consequently, these laboratory tests may represent a patient's coagulation status, but they may not accurately represent the concentration of coagulation factors. Additionally, abnormal coagulation test results in this scenario may trigger the unnecessary use of plasma or cryoprecipitate transfusion.

The decrease of coagulation factors and Fib using clotting time based assays (PT/INR, PTT) following TPE has been described. [1, 2] However, the decrease of protein levels of fibrinogen has not been reported thus far. Therefore, we conducted a prospective investigation of fibrinogen quantity using fibrinogen antigen (FibAg) detection methodology and correlated these results with widely used clot-based Fib tests over the time in patients with AMR of kidney transplants treated with TPE within several days after the biopsy. Additionally, we investigated PT/INR, PTT, factor X chromogenic activity (CFX), ionized calcium (iCa) and platelet count (Plt) over time, and correlated these data with clinical outcome. 


\section{MATERIALS AND METHODS}

We obtained financial support from the University of Michigan Department of Pathology, and the study was approved by the University of Michigan Institutional Review Board. Potential subjects were identified by nephrologists or transfusion medicine physicians, and consent was obtained by participating study coordinators. We recruited adult renal transplant recipients with AMR who were scheduled to receive TPE followed by IVIG per our institutional protocol for participation in the study. Exclusion criteria included children (younger than age 18 years old), patients with elevated liver function tests (reference range at our institution: AST 8-30 IU/L, ALT 7-35 IU/L), pregnant patients, mentally impaired patients and patients who did not provide informed consent. If a patient developed any signs of bleeding such as hematoma or hematuria within 48 hours after the first TPE, the patient was discontinued from study participation and received appropriate treatment. The study was not designed to alter any standard care; therefore, the laboratory test results only for the study purposes were blinded to treating physicians and the study team.

\section{TPE procedure}

Patients received TPE between 3 to 6 times depending on the patient's donor specific antibody level measured by Luminex-based technology (One Lambda, Thermo-Fisher Scientific, Inc., Canoga Park, CA) as mean fluorescence intensity (MFI). Each procedure was performed with one plasma volume exchange, and the replacement fluid consisted of 5\% albumin only or 5\% albumin and plasma (up to half plasma volume) when TPE was performed within 5 days of biopsy according to our institutional protocol for AMR treatment of kidney transplant recipients. When plasma was used, the plasma dose was recorded. Citrate (ACD-A) was infused in blood 
draw line during the TPE procedure at the rate of $0.8 \mathrm{~mL} / \mathrm{min} / \mathrm{LTBV}$ (liter of total blood volume) for both albumin and plasma replacement procedures. The amount of citrate was determined by the apheresis machine, Optia or Spectra (both Terumo BCT, Lakewood, CO), with each patient's total blood volume (TBV) calculated based on sex, height and weight to meet the set infusion rate of $0.8 \mathrm{~mL} / \mathrm{min} / \mathrm{LTBV}$. Therefore, the citrate infusion rate in all patients was constant. Calcium was added to all albumin bottle $(1.2 \mathrm{mmol}$ supplementation per $500 \mathrm{~mL})$ for replacement and provided more in normal saline into return line if the patients showed citrate toxicity or when plasma is used as a replacement, and the dose of total calcium supplementation was recorded.

\section{Laboratory testing}

Standard protocol orders for AMR treatment were made by the primary nephrology team. The schedule for laboratory tests followed; Fib and FibAg, PT/INR/PTT, CFX, and iCa, were immediately Pre-TPE, immediately Post-TPE, 1, 3, 6, 9, 24, and 48 hours after the first TPE; Plt was obtained before TPE (within the same day), immediately Post-TPE, and 24 hours after the first TPE. Additional blood draws for all parameters were obtained before $3^{\text {rd }}$ and $4^{\text {th }}$ TPE for the last 3 patients. The acceptable timing of scheduled blood draws except immediately Pre- and Post-TPE was within 15 minutes for the 1 hour and 3 hour time points after the TPE, and within 2 hours thereafter. All blood samples were de-identified and a study identifier was assigned to each subject. Fib, FibAg, PT/INR, PTT, CFX, iCa and Plt results other than the tests performed as a standard care were held by the hematology and chemistry laboratory directors or supervisors to ensure blinding of the results and to avoid alteration of standard treatment due to test results. Those test results were unblinded after the last subject had completed the study. The test results performed as standard care were retrieved from the electronic medical record. 


\section{Clinical data collection}

Each subject's age, sex, pre-procedure use of anticoagulants, clinical, laboratory and TPE procedural data were retrieved from the electronic medical record and recorded. After the last subject completed the study, all bleeding or thrombotic events noted by the primary nephrology team within 1 month after the last TPE procedure were retrieved from the electronic medical record. Information regarding bleeding and blood product transfusion was also obtained when applicable.

\section{Data analysis}

The correlation of Fib and FibAg was analyzed at each time point. The mean Fib/FibAg ratio before TPE (in the absence of any citrate effect) was 1.08, indicating that Fib results in $8 \%$ higher than FibAg. Therefore, all Fib results were normalized (n) by dividing by 1.08 to compensate for the difference of the test methods. All data was also divided into 3 groups, all patients, and patients with and without plasma replacement use depending on the days after the biopsy to see the effect of plasma use on each parameter. Extreme outliers in coagulation test results were considered to be not clinically plausible, and likely due to heparin contamination of samples drawn through central catheters, or soon after the regular anticoagulant dose. Therefore, data that were greater than 2 standard deviations from the mean for each study parameter and also far different from other data in the same patient representing possible anticoagulant contamination (such as PTT $>100$ seconds with a mean of 23 seconds) were excluded from the analysis. 
A linear mixed effects regression model was used to assess changes of each parameter over the time. Specifically, we modeled the parameter as a piecewise linear function of time, with a change point fixed at the Post-TPE time point. Then slopes before and after the change point were tested to determine whether they are different from the Post-TPE time point. Significance is determined if $\mathrm{p}<0.05$. All analyses were conducted using SAS computer software (version 9.4, SAS Institute, Cary, NC).

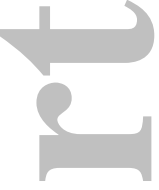

\section{RESULTS}

Eleven patients consented to the study. One patient was found to be pregnant after the consent was obtained and excluded, and one patient withdrew the consent. Thus, the analysis was performed in 9 subjects (A-I). Age ranged from 19 to 67 years old (median 41 years old), 6 males and 3 females (Table 1). Table 1 also shows anticoagulant use, use of plasma as percent of total replacement fluid volume, and the dose of calcium supplementation during TPE. The prescribed anticoagulants were not altered during the course of TPE treatment. One patient on low molecular weight heparin had much lower CFX level only at the Pre-TPE than CFX levels at other time points after TPE (more than $70 \%$ lower); therefore, this data was excluded from analysis. Two data points in PT/INR and two data points in PTT were far above two standard deviations and were excluded from the data analysis due to possible anticoagulant contamination.

Table 2 shows mean and range of test results in each parameter at Pre- and Post-TPE. The mean level change (\%) of normalized Fib (nFib), FibAg, CFX, PT/INR, and PTT based on the Pre-TPE level at each time point are shown in Figure 1A-F. All figures show level change in 3 groups; all patients, patients with plasma replacement and without plasma replacement. 
The $n$ Fib/FibAg ratio at each time point in each patient is shown in Table 3. The mean change of nFib/FibAg ratio at each time point based on Pre-TPE level in the same 3 groups above is shown in Figure 2. Overall, nFib/FibAg ratio decreased at Post-TPE and returned to Pre-TPE level in 6 hours. However, this ratio returned to Pre-TPE level in 3 hours after TPE in patient group with plasma replacement; and the ratio did not return to pre-TPE level in patient group without plasma replacement although returning slope was observed toward 6 hour time point.

Statistical significance ( $p$-value) of level decrease or increase before and after Post-TPE time point in all patients is shown above the each graph in Figure 1 and 2. P-values of the slope before and after the Post-TPE in each parameter in 3 groups (all patients, patients with and without plasma replacement) are shown in Supplement Table 1. Overall, nFib, FibAg and CFX showed statistically significant decrease after the TPE, but only nFib showed significance in returning slope to the Pre-TPE level. Increasing slope toward Post-TPE and returning slope were statistically significant in PT/INR, but not significant in PTT. Decrease of nFib/FibAg ratio toward Post-TPE was not statistically significant; however, the return was significant.

Between 2 groups, patients treated with and without plasma replacement, the differences of level change were not statistically significant for nFib, FibAg, nFib/FibAg ratio and CFX, but were statistically significant for PT and PTT (Table 4).

Ionized Ca was from $0.97-1.45 \mathrm{mmol} / \mathrm{L}$ with one exception of $0.3 \mathrm{mmol} / \mathrm{L}$ with $\mathrm{INR}$ of 1.2 at Post-TPE in one patient whose iCa returned to $1.32 \mathrm{mmol} / \mathrm{L}$ at 1 hour Post-TPE (Figure 3). Plt ranged from 105 to $305 \mathrm{~K} / \mu \mathrm{L}$.

Percentage of nFib, FibAg, CFX and Plt based on the Pre-TPE level before $2^{\text {nd }}$ (48 hours), $3^{\text {rd }}$ (96 hours) and $4^{\text {th }}$ (144 hours) TPE from 3 patients who were not on any anticoagulant is 
shown in Table 5. No trending decrease was observed in any of the parameter after the second TPE.

Two patients had bleeding episodes after TPE treatment. One patient on warfarin had bloody bowel movements from existing non-thrombosed external hemorrhoids observed by flexible sigmoidoscopy 6 days after the first TPE, a day after the 3rd TPE procedure. This patient received a transfusion of one unit of plasma, and iron supplementation for Hgb 9.0 g/dL. The patient's hemoglobin remained stable with no further bleeding. The other patient had heavy menses 7 days after the last TPE, in the setting of irregular menstrual cycles and possible leiomyoma. This patient did not receive any transfusion.

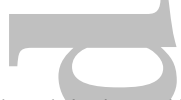

\section{DISCUSSION}

The higher likelihood of bleeding complication following invasive procedures in patients receiving TPE is a concern in patients who are undergoing AMR treatment. Percutaneous renal biopsy is a well-established procedure and the occurrence rate of bleeding complications such as hematoma and hematuria ranges from $1.0 \%$ to $33.3 \%$ ( $>90 \%$ clinically silent) and from 3.0 to 9.9\%, respectively in literatures. [3-6] TPE may increase the risk of bleeding by removal of coagulation factors, or by the anticoagulant effect of citrate. One plasma volume TPE decreases plasma protein levels by approximately 65\%. [7] One study showed that Fib decreased by average $42 \%$ and other coagulation factors decreased between $22.7 \%$ and $55.3 \%$ immediately after the TPE, however, the absolute concentrations remained within or only slightly below the reference range [1]. At our institution, at least $10 \mathrm{~mL} / \mathrm{Kg}$ of plasma is used as a replacement fluid for TPE to add coagulation factors in patients within 5 days of their biopsies. Thus, increased bleeding risk due to depletion of coagulation factors would not generally be expected. 
Calcium is one of co-factors in the coagulation cascade and is required by seven Vitamin$\mathrm{K}$ dependent coagulation factors. Citrate used during TPE binds to circulating divalent cations, including $\mathrm{Ca}^{2+}$ and $\mathrm{Mg}^{2+}$, in the blood, leading to a decrease in 10-15\% of iCa concentration [8]. This reduction in iCa slows the process of coagulation cascade and reduces platelet aggregation/fibrinogen binding [9], and prevents clotting in the extracorporeal circuit during TPE. However, reduced iCa may exacerbate the effect of TPE-related decrease of coagulation factors, leading to a coagulopathic status and increased susceptibility to bleeding following the TPE until the citrate effect is diminished by normal metabolism. Strauss calculated that the $15-24 \mathrm{mmol} / \mathrm{L}$ citrate levels achieved within cell separation circuits during a typical apheresis procedure would lower the iCa below $0.2-0.3 \mathrm{mmol} / \mathrm{L}$ [10]. Since all our participants received $\mathrm{Ca}$ supplementation into their return lines, the Post-TPE iCa value in most patients was only slightly decreased (figure 3). However, the dose of Ca supplementation was not constant in each patient with variable $\mathrm{Ca}$ infusion rates during each procedures, the distribution and elimination of $\mathrm{Ca}$ may not be constant in our patients who had variable degrees of renal insufficiency, the duration of each TPE procedure was not constant, and additional citrate from plasma was administered when plasma was used for replacement. Therefore, iCa data may not be meaningful to evaluate citrate level at the Post-TPE time point. In fact, nFib/FibAg ratio, nFib levels or INR did not show correlation with iCa levels in 6 patients for whom iCa data was available at the Post-TPE time point (Supplement Figure 1). Notably, iCa level in one patient at the Post-TPE was 0.3 $\mathrm{mmol} / \mathrm{L}$ with an INR value of 1.2. This patient received albumin replacement at first with constant $\mathrm{Ca}$ supplementation in albumin bottle and plasma replacement at the end of TPE with some additional Ca supplement in normal saline (total11.2 mmol Ca supplementation). This case most likely suggests that the citrate dose was enough to decrease $\mathrm{iCa}$, but $\mathrm{Ca}$ 
supplementation at the end of TPE was insufficient to completely overcome this effect.

Furthermore, the INR level at the Post-TPE time point did not reflect low iCa level, suggesting that temporally low iCa level may not be clinically important.

Fibrinogen is most commonly measured by the fibrinogen activity test, by determining the clotting time of diluted plasma after the addition of thrombin. The clotting endpoint is compared to a standard reference curve and the fibrinogen concentration is determined. With the presence of citrate, the clotting time will be prolonged, and thus the Fib result will be lower. On the other hand, the FibAg test is performed by radial immunodiffusion and detects fibrinogen antigen-antibody complex using an agarose gel containing an antibody specific for fibrinogen. Antigen-antibody complexes will form a precipitin ring and the ring size is proportional to the antigen concentration. Thus, the results of this test are not altered by the presence of citrate. In our limited number of participants, the Fib result was on average $11 \%$ lower than the FibAg test result immediately after TPE, and returned close to equivalent in 6 hours (Table 3, Figure 2). This data suggests a citrate effect on fibrinogen activity measurements, although the changes of the nFib/FibAg ratio at any time point ( $p>0.7125$, not data shown) and the decrease of the $n$ Fib/FibAg ratio ( $p=0.3738$, Figure 2 ) were not statistically significant. Similarly, any tests reliant on calcium may be affected by citrate, therefore, CFX may be falsely lower, and PT/INR and PTT may be falsely higher up to 6 hours after TPE. We found that PTT also returned to the Pre-TPE level at the 6 hour time point overall.

The lowest Fib, FibAg, CFX, Plt and iCa were 110mg/L, 134mg/L, 40\%, $107 \mathrm{~K} / \mu \mathrm{L}$, and $0.97 \mathrm{mmol} / \mathrm{L}$ (except for one patient discussed above) respectively (at the Post-TPE time point), which should be sufficient to maintain hemostasis. However, all nFib, FibAg and CFX levels did not return to Pre-TPE level within 48 hours (Figure 1), which may raise a possibility of 
further decreases with frequent TPE. The percent of nFib, FibAg, CFX and Plt based on the PreTPE levels prior to $2^{\text {nd }}, 3^{\text {rd }}$ and $4^{\text {th }}$ TPE did not show progressive decline (Table 5 ) in data from 3 patients without anticoagulants. One patient among these 3 patients received plasma replacement only in the first TPE. These data were not affected by citrate because subsequent TPEs were performed at around 48 hours after the each TPE, given that the citrate effect appears to last for 6 hours from our study. This observation suggests that coagulation factors decreased by TPE return to their pre-TPE level in 2 days after the second TPE. Therefore, plasma or cryoprecipitate transfusion is not required even with multiple procedures when TEP procedures are performed every other day or less frequently. A previous report had similar finding. [1] Blasi A. et al. showed that fibrinogen levels before first TPE and second TPE (one or two days after the first TPE) were similar albeit with reduced clot firmness that was without clinical implications [11]. However, our results are based on a limited number of patients and a larger study is necessary to confirm this observation, especially since we found that fibrinogen recovery was slightly delayed relative to CFX.

When 2 groups, patients with and without plasma replacement use, were compared, nFib, FibAg, nFib/FibAg ratio and CFX did not show statistically significant differences (Table 4), which may also suggest plasma use for replacement may not improve coagulation status after TPE procedure under the effect of citrate. However, PT and PTT showed statistically significant difference in these 2 groups, with the level increase at Post-TPE greater in the patient group without plasma replacement. This may be due to PT and PTT being relatively sensitive to coagulation factor levels below 50\%; these test results might be affected by clinically nonsignificant decrease of coagulation factors to between 20 and $50 \%$ of reference range after TPE. Coagulation factor VIII may require more than $30 \%$ of normal for normal hemostasis, while 
other coagulation factors likely provide sufficient hemostasis at $10-20 \%$ of normal. [12] However, our study showed that the lowest CFX was 40\% (32.8\% of Pre-TPE level) even with citrate effect. Therefore, all coagulation factors should be more than $30 \%$ of normal unless PreTPE levels were also lower. Thus, PT/INR, and PTT may be increased more in patients without plasma use due to some coagulation factors $<50 \%$ of normal, although the amount of coagulation factors are likely sufficient for hemostasis in both groups.

Limitations of this study are the small number of participants with some missing data, and the potential confounding effect of systemic anticoagulation in some patients, and the possible contamination of some samples by heparin used for central line flushing. Therefore, the data from only patients who were not on anticoagulants was also analyzed. Normalized Fib/FibAg ratio and CFX over the time for those patients demonstrated the similar results between all patients and patients without anticoagulants (Supplement Figure 2). Therefore, overall analysis was performed using all patients' data.

Lastly, two patients had bleeding episodes from sites other than kidney biopsy within several days after TPE. However, these episodes were unlikely related to either citrate or TPE effect, but rather related to the patients' underlying conditions.

In summary, we found a difference between nFib and FibAg levels up to 6 hours after TPE, although statistically not significant, which suggests a persistent citrate effect for this period. Similarly, any tests reliant on calcium may be affected by citrate; CFX may be falsely lower, and PT/INR and PTT may be falsely higher up to 6 hours after TPE. Both FibAg and CFX did not return to Pre-TPE level in 48 hours after the first TPE procedure, however, the levels of FibAg and CFX before the subsequent TPE did not show a tendency for progressive decline. In addition, FibAg, CFX and Plt showed sufficient levels to maintain coagulation status 
at all-time points observed, even in the presence of a putative citrate effect. Therefore, blood component transfusion is not necessary for coagulation factor replacement when TPE is performed every other day or less frequently, even though the patients can be coagulopathic due to citrate effect up to 6 hours after TPE. Furthermore, nFib, FibAg and CFX results did not show a statistically significant difference between patients with and without plasma replacement use, which may suggest plasma use as a replacement fluid up to $45 \%$ of total replacement volume may not have any effect on coagulation status. A larger study may be necessary to confirm these findings.
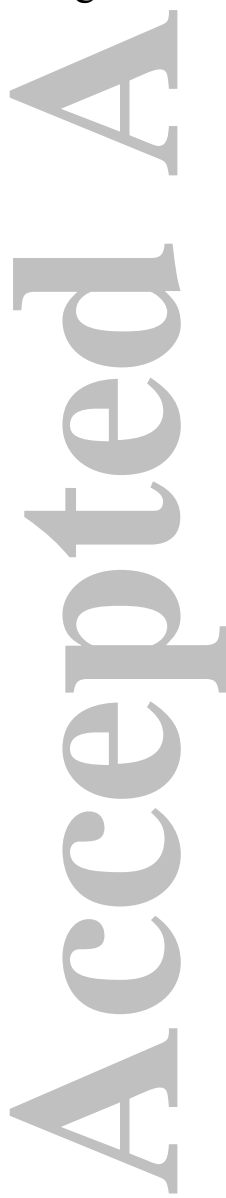


\section{ACKNOWLEDGEMENTS}

We thank research coordinators in transplant -surgery, apheresis nurses, chemistry and hematology laboratory supervisors and technologists at University of Michigan for their contribution to this study. We also thank University of Michigan Pathology for financial support on lab tests and research coordinators' fees.
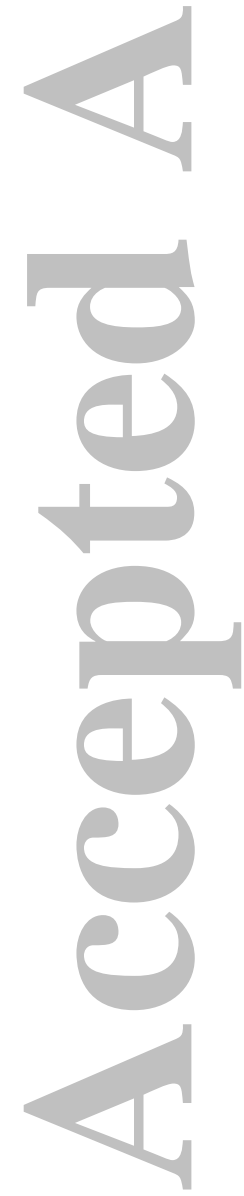


\section{REFERENCES}

1. Volkin RL, Starz TW, Winkelstein A, Shadduck RK, Lewis JH, Hasiba U, Spero JA. Changes in coagulation factors, complement, immunoglobulins, and immune complex concentrations with plasma exchange. Transfusion 1982;22(1): 54-8.

2. Wood L and Jacobs P. The effect of serial therapeutic plasmapheresis on platelet count, coagulation factors, plasma immunoglobulin, and complement levels. J Clin Apher 1986;3(2): 124-8.

3. Gainza FJ, Minguela I, Lopex-Vidaur I, Ruiz LM, Lampreabe I. Evaluation of complications due to percutaneous renal biopsy in allografts and native kidneys with color-coded Doppler sonography. Clin Nephrol 1995;43(5): 303-8.

4. Manno C, Strippoli GF, Amesano L, Bonifati C, Campobasso N, Gesualdo L, Schena FP. Predictors of bleeding complications in percutaneous ultrasound-guided renal biopsy. Kidney Int 2004;66(4): 1570-7.

5. Parrish AE. Complications of percutaneous renal biopsy: a review of 37 years' experience. Clin Nephrol 1992;38(3): 135-41.

6. Whittier WL and Korbet SM. Renal biopsy: update. Curr Opin Nephrol Hypertens $2004 ; 13(6): 661-5$

7. Davenport RD. Therapeutic Aspheresis. Technical manual. $18^{\text {th }}$ ed. Bethesda (MD): AABB; 2014 p.646

8. Haddad, S, Leitman SF, Wesley RA, Cecco S, Yau YY, Starling J, Rehak NN, Bolan CD. Placebo-controlled study of intravenous magnesium supplementation during largevolume leukapheresis in healthy allogeneic donors. Transfusion 2005;45(6):934-44 
9. Kingston JK, Bayly WM, Sellon DC, Meyers KM, Wardrop KJ. Effects of sodium citrate, low molecular weight heparin, and prostaglandin E1 on aggregation, fibrinogen binding, and enumeration of equine platelets. Am J Vet Res. 2001;62(4):547-54

10. Strauss RG. Mechanisms of Adverse Effects During Hemapheresis. J Clin Apher $1996 ; 11(3): 160-4$

11. Blasi A, Cid J, Beltran J, Raura P, Balust J, Lozano M. Coagulation profile after plasma (1)

exchange using albumin as a replacement solution measured by theromboelastometry.

Vox Sang 2016;110(2):159-65

12. Nester T, Jain S, Poisson J. Hemotherapy Decisions and Their outcome. Technical manual. $18^{\text {th }}$ ed. Bethesda (MD): AABB; 2014 p.521

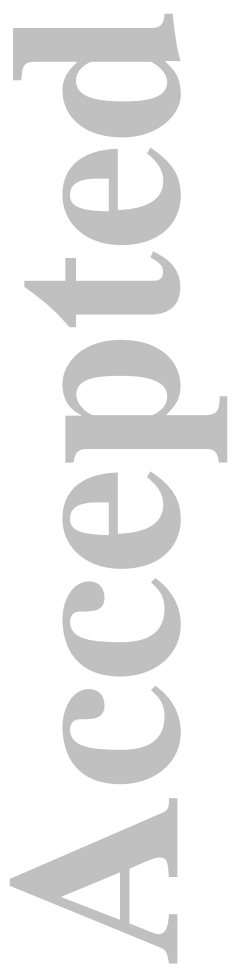


Table 1: Demographics, TPE* procedure data and anticoagulant use

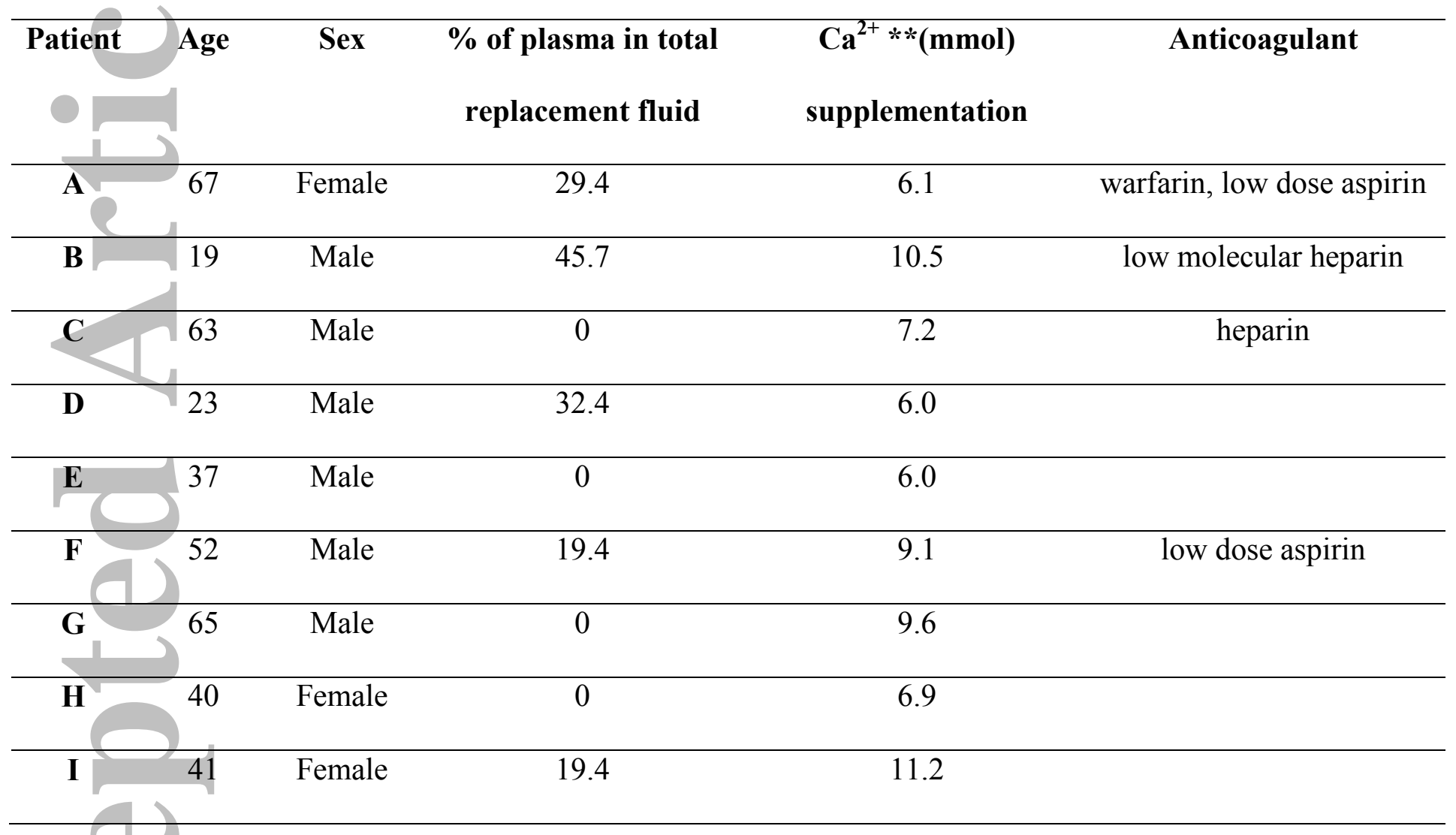

$\mathrm{TPE}^{*}$ : therapeutic plasma exchange, $\mathrm{Ca}^{* *}$ : calcium 
Table 2: Mean and range value of each parameter at Pre- and Post-TPE time points

\begin{tabular}{lcc}
\hline Parameter & Pre-TPE* & Post-TPE \\
\hline Fib $^{\dagger}$ (mg/dL) & $412.0(339-465)$ & $187.0(110-293)$ \\
\hline FibAg $^{\dagger}$ (mg/dL) & $392.25(326-513)$ & $194.89(135-235)$ \\
\hline PT (seconds) & $11.4(10.6-13.8)$ & $13.53(11.4-16.4)$ \\
\hline INR & $1.09(1.0-1.4)$ & $1.31(1.1-1.6)$ \\
\hline PTT (seconds) & $26.04(21.4-33.3)$ & $29.76(25.5-34.4)$ \\
\hline CFX & & \\
\hline
\end{tabular}

TPE*: therapeutic plasma exchange, Fib $^{\dagger}:$ fibrinogen activity test result, FibAg $^{\dagger}$ : fibrinogen antigen test result, $\mathrm{CFX}^{\S}$ : coagulation factor $\mathrm{X}$ chromogenic activity test result 
Table 3: $\mathrm{nFib}^{\dagger} / \mathrm{FibAg}^{\ddagger}$ ratio at each time point in each patient

\begin{tabular}{lllllllll}
\hline Patient & Pre & Post & 1 hour & 3 hour & 6 hour & 9 hour & 24 hour & 48 hour \\
\hline A & 1.02 & 0.99 & 0.93 & & & & 1.09 & 1.22 \\
\hline B & & 0.83 & 0.89 & 0.70 & & & 1.04 & \\
\hline C & 1.17 & 0.88 & 0.89 & 0.79 & 0.79 & 0.81 & 0.76 & 0.89 \\
\hline D & 0.99 & 0.96 & 0.71 & 0.82 & 0.99 & 0.97 & 1.15 & 0.88 \\
\hline E & 1.22 & 0.96 & 1.12 & 1.26 & 1.36 & & 1.00 & 1.26 \\
\hline F & 1.07 & 1.22 & 1.21 & 1.35 & 1.30 & 1.21 & 1.32 & 0.83 \\
\hline G & 0.92 & 0.76 & 0.76 & 0.88 & 0.95 & 0.95 & 1.06 & 0.82 \\
\hline H & 0.61 & 0.43 & 0.56 & 0.42 & 0.61 & 1.06 & 0.82 & 1.24 \\
\hline I & 0.97 & 0.99 & 1.02 & 1.13 & 1.10 & & 1.20 & 1.02 \\
\hline Mean & 1.00 & 0.89 & 0.90 & 0.92 & 1.01 & 1.00 & 1.05 & 1.02 \\
\hline
\end{tabular}

$\mathrm{nFib}^{\dagger}$ : normalized fibrinogen activity test result, FibAg ${ }^{\ddagger}$ : fibrinogen antigen test result 
Table 4: Statistical significance (p-value) of difference between with and without plasma replacement before and after Post-TPE* time point

\section{A: Difference before Post-TPE}

\begin{tabular}{|c|c|c|}
\hline Change & Parameter & p-value \\
\hline \multirow[t]{4}{*}{ Decreasing } & nFib $^{\dagger}$ & 0.2122 \\
\hline & $\operatorname{FibAg}^{\ddagger}$ & 0.6144 \\
\hline & nFib/FibAg ratio & 0.079 \\
\hline & $\mathrm{CFX}^{\S}$ & 0.0952 \\
\hline \multirow[t]{3}{*}{ Increasing } & PT & 0.0336 \\
\hline & INR & 0.061 \\
\hline & PTT & 0.0187 \\
\hline
\end{tabular}

3B: Difference after Post-TPE

\begin{tabular}{|c|c|c|}
\hline Change & Parameter & p-value \\
\hline \multirow[t]{4}{*}{ Increasing } & nFib & 0.1444 \\
\hline & FibAg & 0.3708 \\
\hline & nFib/FibAg ratio & 0.1252 \\
\hline & CFX & 0.1215 \\
\hline \multirow[t]{3}{*}{ Decreasing } & PT & 0.0127 \\
\hline & INR & 0.0219 \\
\hline & PTT & 0.0041 \\
\hline
\end{tabular}


TPE*: therapeutic plasma exchange, $\mathrm{nFib}^{\dagger}$ : normalized fibrinogen activity test result, FibAg*: fibrinogen antigen test result, $\mathrm{CFX}^{\S}$ : coagulation factor $\mathrm{X}$ chromogenic activity test result

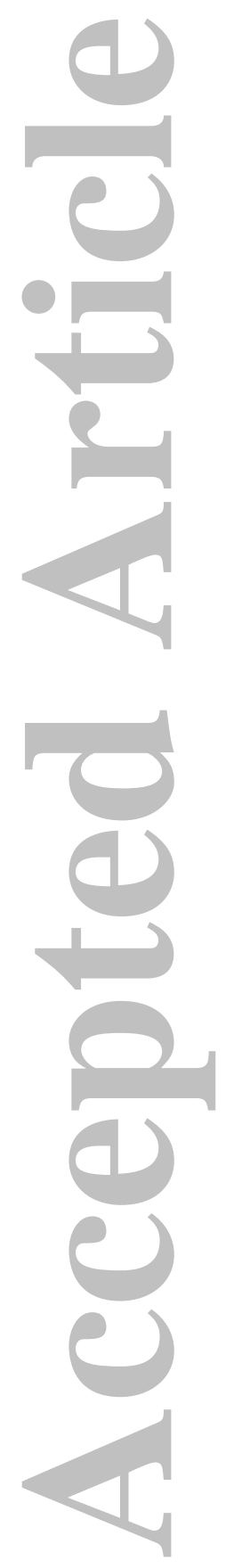

ScholarOne, 375 Greenbrier Drive, Charlottesville, VA, 229011 (434) 964-4100

This article is protected by copyright. All rights reserved. 


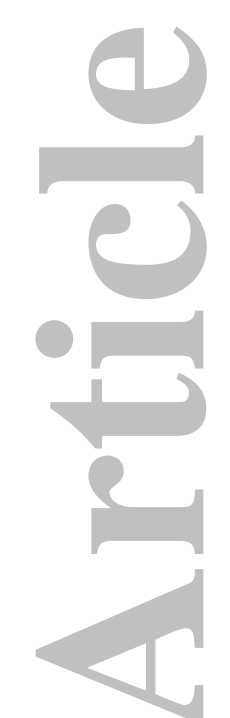

Table 5: Mean \% of coagulation factors based on Pre-TPE level prior to subsequent TPE on 3 patients without anticoagulants

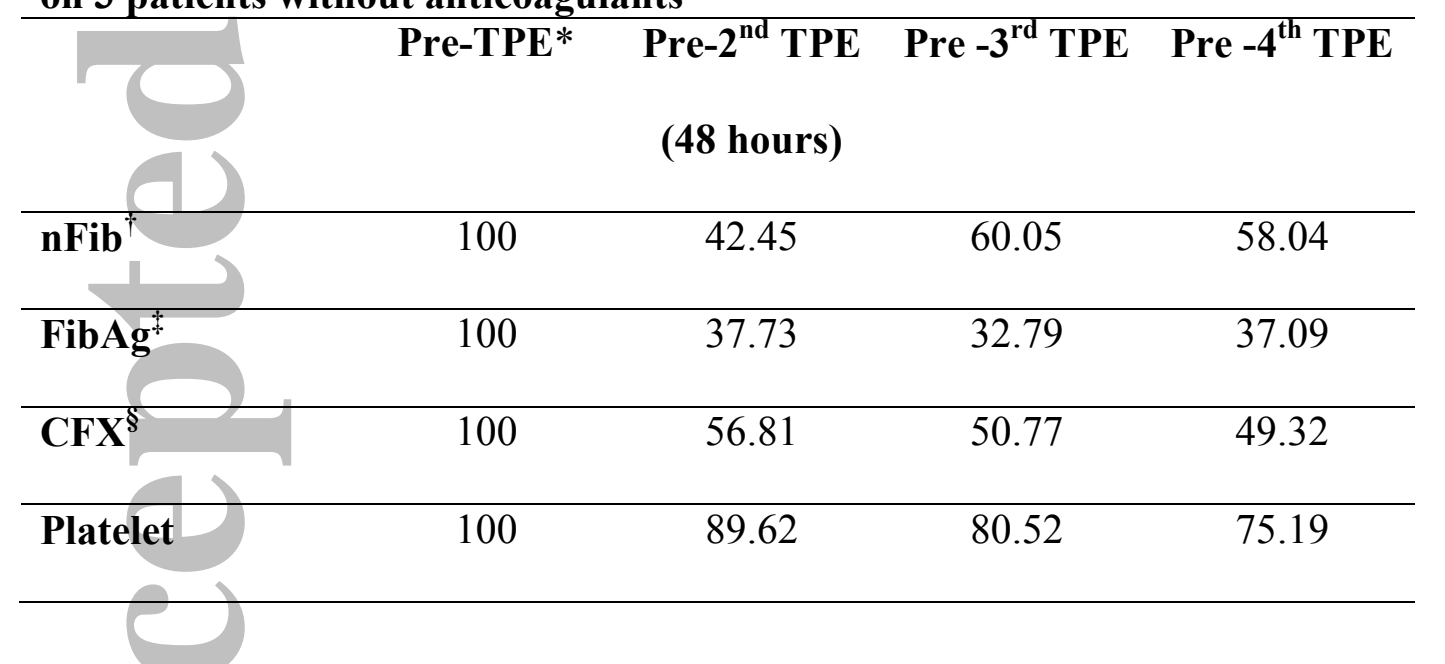

$\mathrm{TPE}^{*}:$ therapeutic plasma exchange, $\mathrm{nFib}^{\dagger}$ : normalized fibrinogen activity test result, FibAg*:

fibrinogen antigen test result, $\mathrm{CFX}^{\S}$ : coagulation factor $\mathrm{X}$ chromogenic activity test result 
Figure 1: The mean level change (\%) based on Pre-TPE* level at each time point

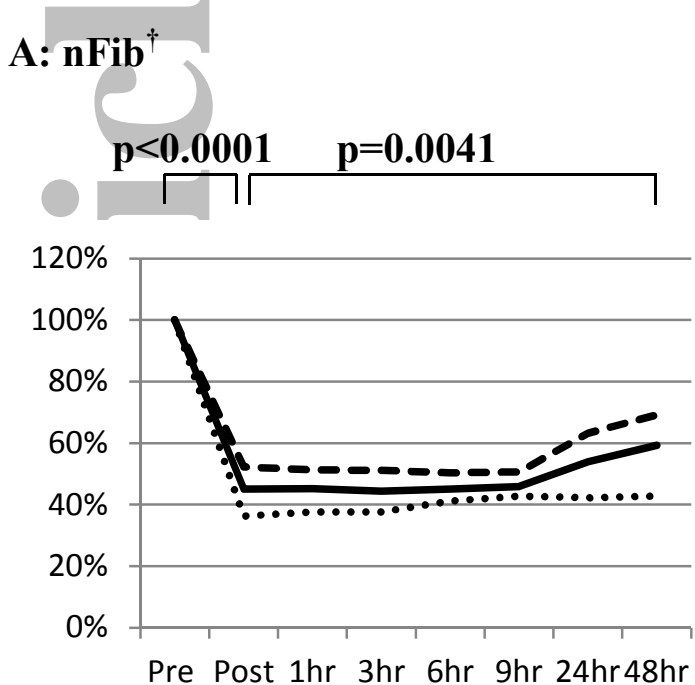

D: PT
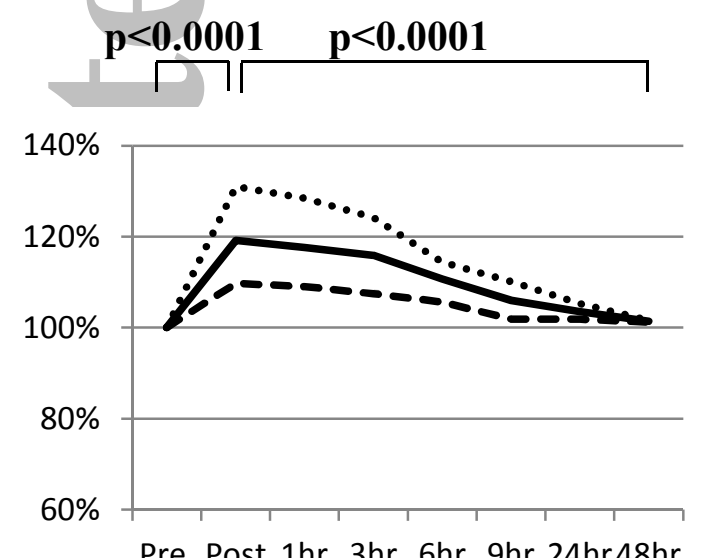

Pre Post $1 \mathrm{hr} 3 \mathrm{hr} 6 \mathrm{hr} 9 \mathrm{hr} 24 \mathrm{hr} 48 \mathrm{hr}$

\section{B: FibAg $g^{\ddagger}$}

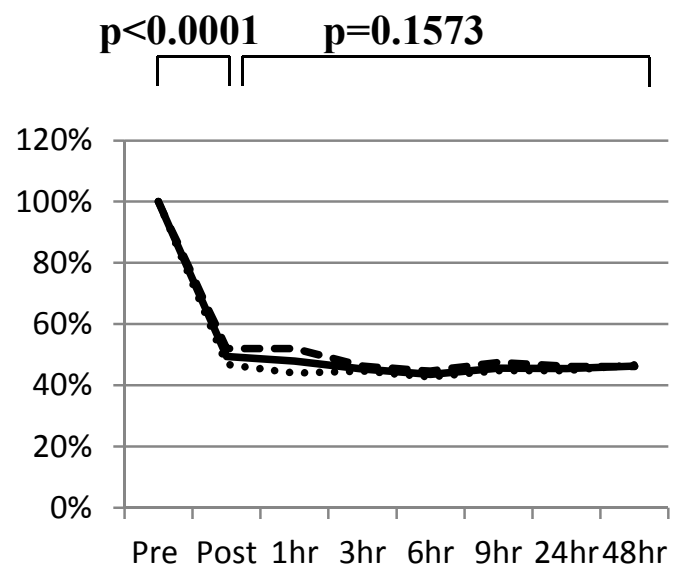

\section{E: INR}
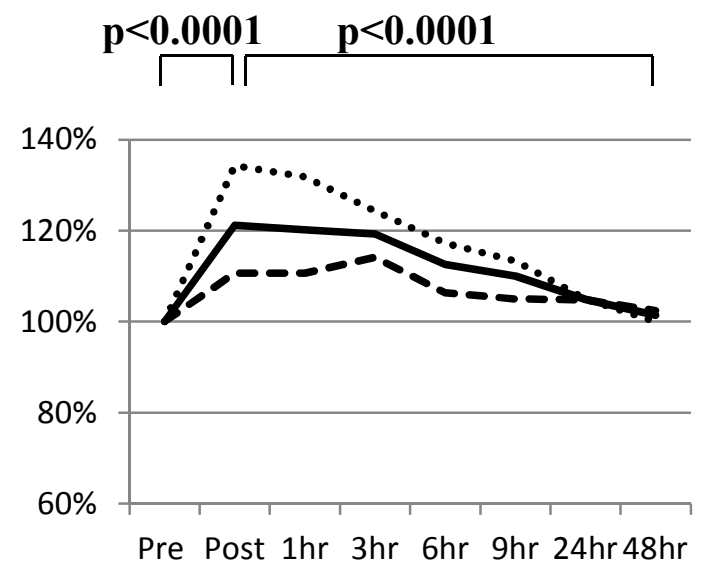

$\mathrm{C}: \mathbf{C F X}^{\S}$

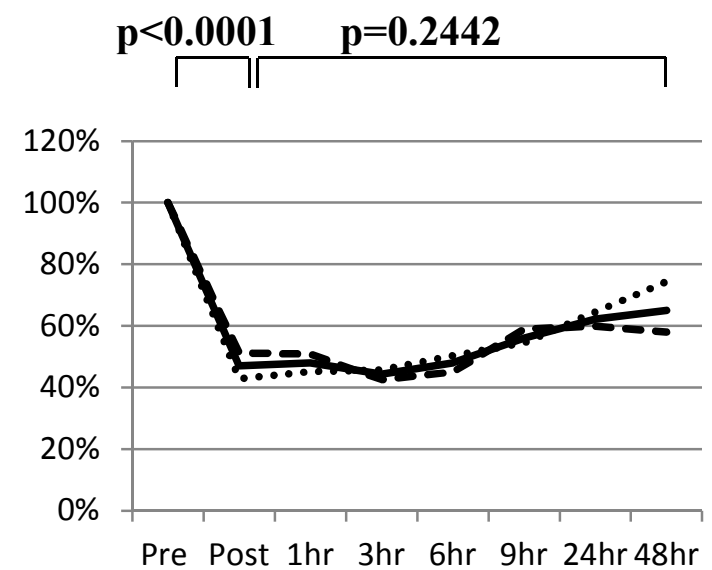

\section{F: PTT}

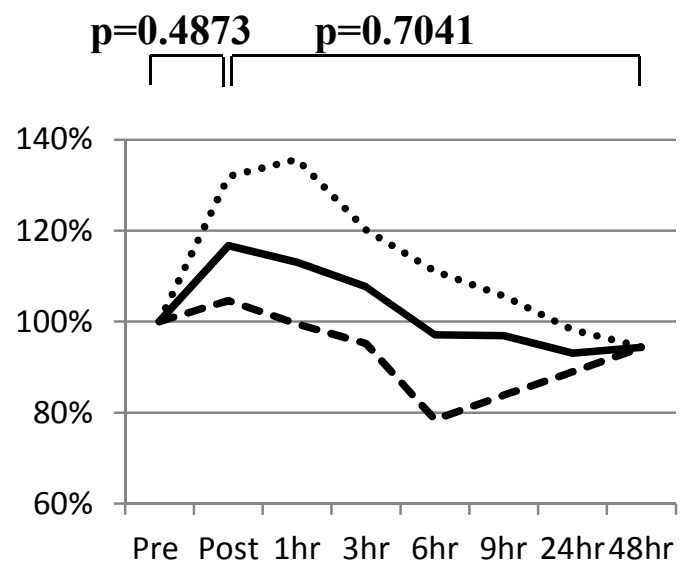

- With plasma replacement

..... Without plasma replacement 
Figure 2: The mean change of $\mathrm{nFib}^{\dagger} / \mathrm{FibAg}^{\ddagger}$ ratio (\%) based on Pre-TPE* level at each time point

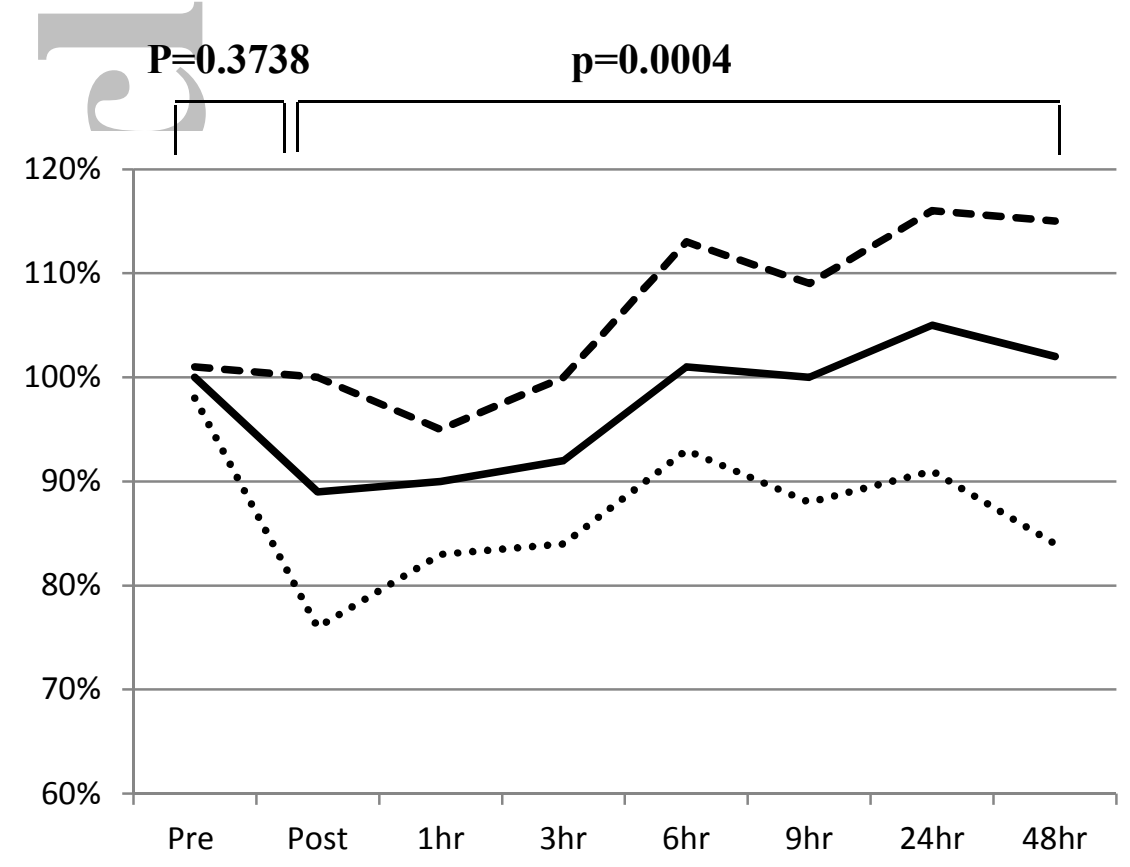

All patients

- With plasma replacement

-... Without plasma replacement

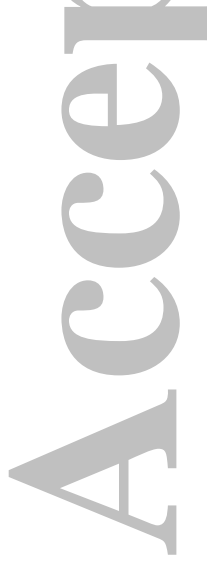


Figure 3: Ionized Calcium values at each time point in each patient

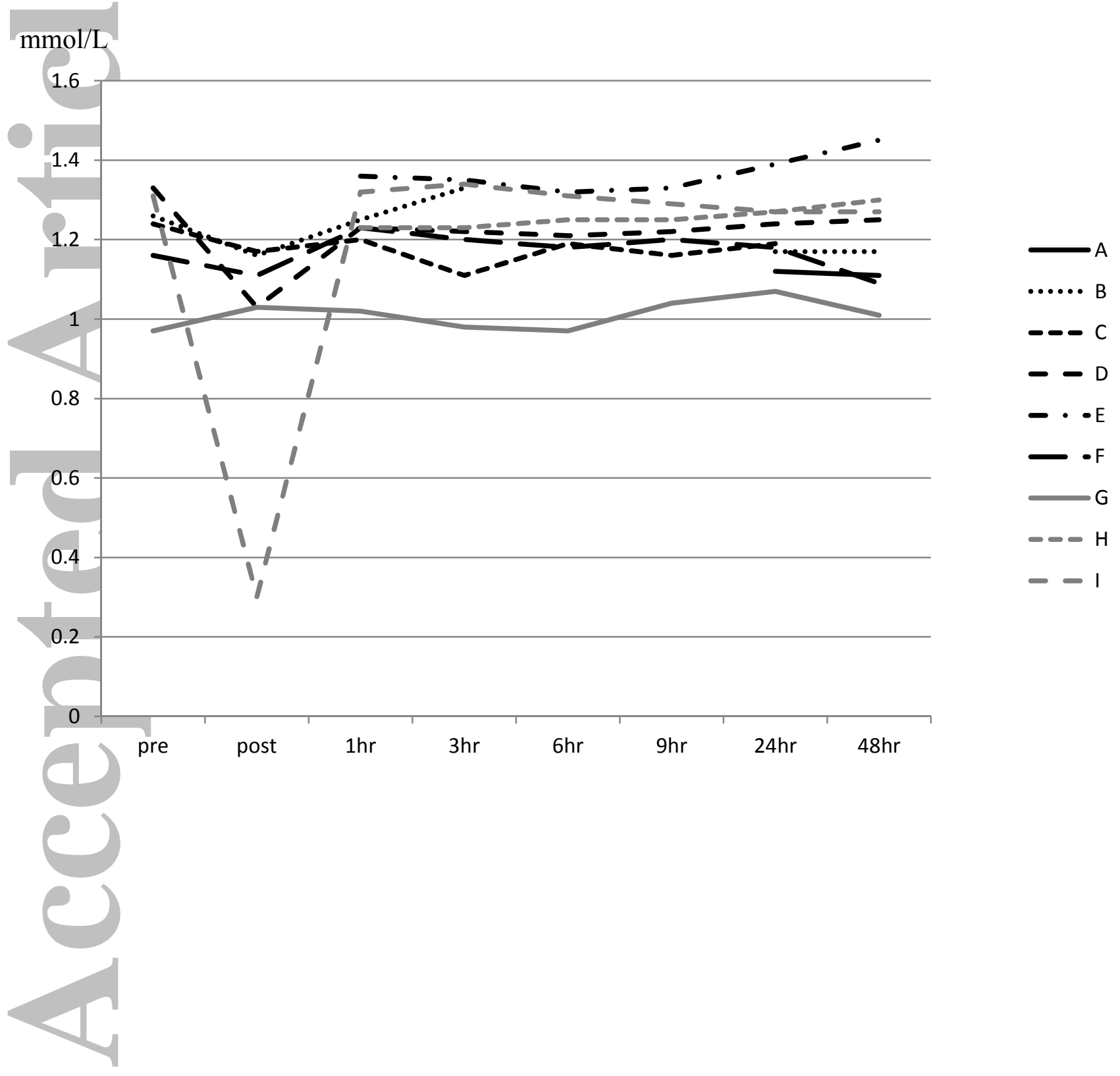




\section{Figure Legends}

Figure 1: The mean level change (\%) based on Pre-TPE* (Therapeutic plasma exchange) level at each time point. A) $\mathrm{nFib}^{\dagger}$ : Normalized fibrinogen activity test result. B) FibAg ${ }^{\ddagger}$ : Fibrinogen antigen test result. C) $\mathrm{CFX}^{\S}$ : Coagulation factor $\mathrm{X}$ chromogenic activity test result. D) PT. E) INR. F) PTT. All data is shown in 3 groups, all patients ( $(\boldsymbol{)})$, patients with plasma

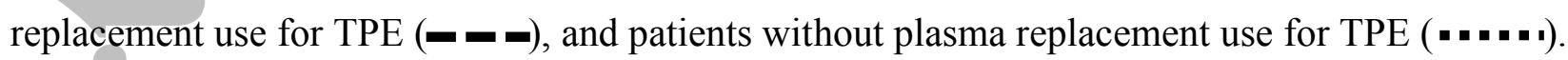
Statistical significance ( $\mathrm{p}$-value) of level change before and after Post-TPE time point in all patients (both patients with and without plasma replacement) is shown above the each graph. Pvalue on the left above each graph is level decrease/increase before Post-TPE and that on the right is level increase/decrease after Post-TPE. Statistical significance is determined if $\mathrm{p}<0.05$.

Figure 2: The mean change of $\mathrm{nFib}^{\dagger} / \mathrm{FibAg}^{*}$ ratio (\%) based on Pre-TPE* level at each time point. The data is also shown in 3 groups as same as Figure 1. P-value is also shown as same as Figure 1.

Figure 3: Ionized Calcium values at each time point in each patient. The data of each patient is shown in different line type and/or color. 
Supplement Table 1: Statistical significance (p-value) of level change before and after Post-

TPE time point

S1A: Level change before Post-TPE*

\begin{tabular}{|c|c|c|c|c|}
\hline \multirow[t]{2}{*}{ Change } & \multirow[t]{2}{*}{$\underline{\text { Parameter }}$} & \multicolumn{3}{|c|}{ p-value } \\
\hline & & All & With plasma & Without plasma \\
\hline \multirow[t]{4}{*}{ Decreasing } & $\underline{\mathrm{nFib}^{\dagger}}$ & $<0.0001$ & $<0.0001$ & $<0.0001$ \\
\hline & FibAg" & $<0.0001$ & $\leq 0.0001$ & $<0.0001$ \\
\hline & $\underline{\text { nFib/FibAg ratio }}$ & $\underline{0.3738}$ & $\underline{0.3901}$ & $\underline{0.1259}$ \\
\hline & $\mathrm{CFX}^{\S}$ & $\leq 0.0001$ & $\leq 0.0001$ & $\leq 0.0001$ \\
\hline \multirow[t]{3}{*}{ Increasing } & $\underline{\mathrm{PT}}$ & $<0.0001$ & $\underline{0.0031}$ & $\underline{0.0002}$ \\
\hline & INR & $\leq 0.0001$ & $\underline{0.0045}$ & $\underline{0.0003}$ \\
\hline & $\underline{\text { PTT }}$ & 0.4873 & $\underline{0.3078}$ & $\underline{0.0288}$ \\
\hline
\end{tabular}

S1B: Level change after Post-TPE

\begin{tabular}{|c|c|c|c|c|}
\hline \multirow[t]{2}{*}{ Change } & \multirow[t]{2}{*}{ Parameter } & \multicolumn{3}{|c|}{ p-value } \\
\hline & & All & $\underline{\text { With plasma }}$ & Without plasma \\
\hline \multirow[t]{4}{*}{ Increasing } & $\underline{\text { nFib }}$ & $\underline{0.0041}$ & $\underline{0.2981}$ & $<0.0001$ \\
\hline & FibAg & $\underline{0.1573}$ & $\underline{0.1241}$ & $\underline{0.7683}$ \\
\hline & nFib/FibAg ratio & $\underline{0.0004}$ & $\underline{0.04}$ & $\underline{0.0043}$ \\
\hline & $\underline{\text { CFX }}$ & $\underline{0.2442}$ & $\underline{0.9566}$ & $\underline{0.0051}$ \\
\hline \multirow[t]{3}{*}{ Decreasing } & $\underline{\mathrm{PT}}$ & $<0.0001$ & $\underline{0.0027}$ & $\leq 0.0001$ \\
\hline & INR & $\leq 0.0001$ & $\underline{0.0105}$ & 0.0001 \\
\hline & PTT & 0.7041 & 0.1481 & 0.0099 \\
\hline
\end{tabular}




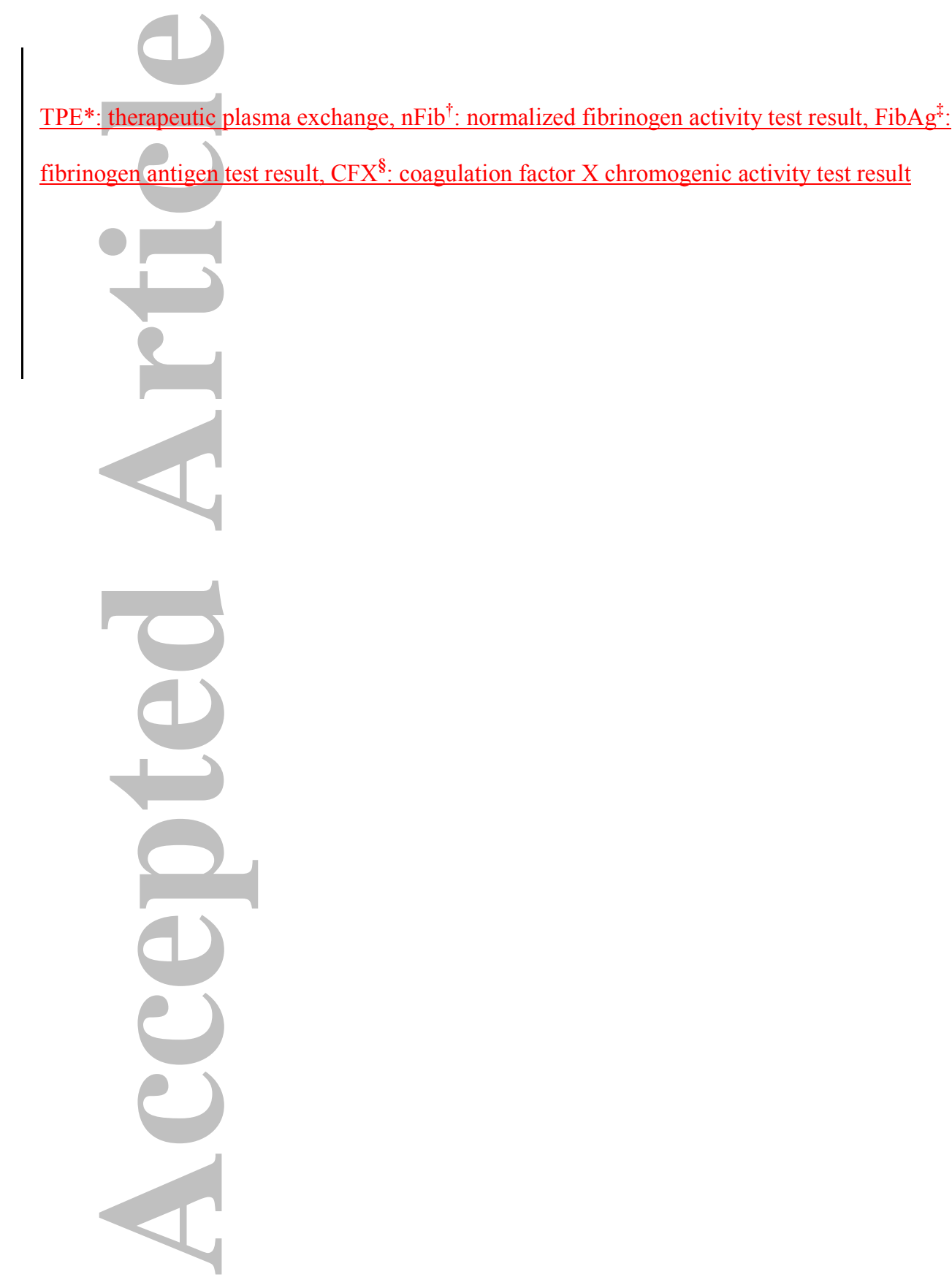

ScholarOne, 375 Greenbrier Drive, Charlottesville, VA, 229011 (434) 964-4100 This article is protected by copyright. All rights reserved. 
Supplement Figure 1: nFib $^{\dagger} /$ FibAg $^{\ddagger}$ ratio, nFib, INR and iCa. at Post-TPE* time point (iCa: $\bar{m} \overline{m o l} / \mathrm{L}, \bar{n}$ Fib/FibAè rātiō, INR) $(\overline{\mathrm{n} F i b} \overline{\mathrm{m}} \mathrm{g} / \mathrm{dL})$
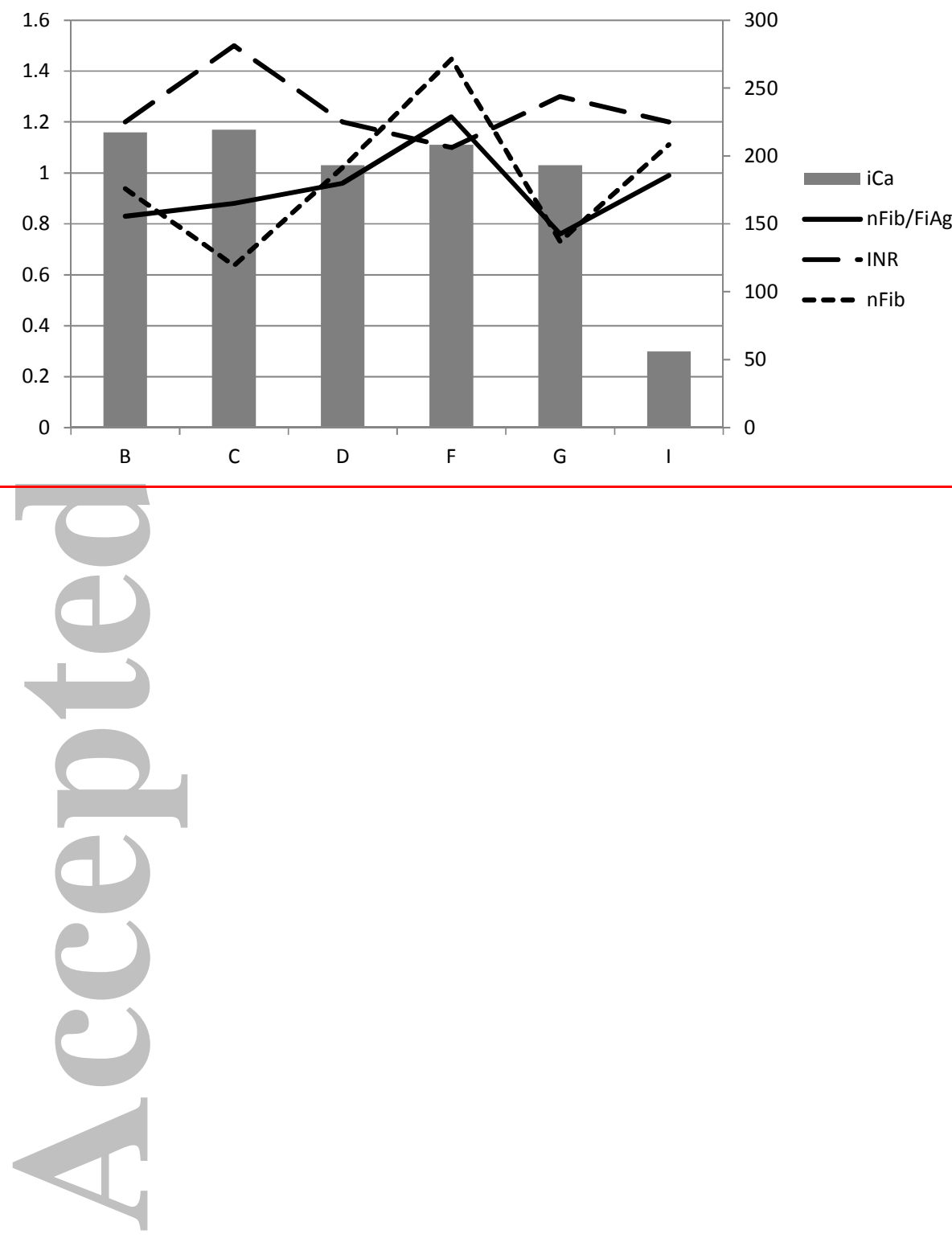

Formatted: Superscript

Formatted: Font: (Default) Times New Roman, 12 pt, Bold

Formatted: Font: (Default) Times New Roman, 12 pt

Formatted: Font: (Default) Times New Roman, 12 pt

Formatted: Space After: 10 pt, Line spacing: Multiple $1.15 \mathrm{li}$ 
Supplement Figure 21: The mean level change (\%) based on Pre-TPE* level at each time point on patients without anticoagulants

| A: Fib $^{\dagger} /$ FibAg ratio $^{*}$

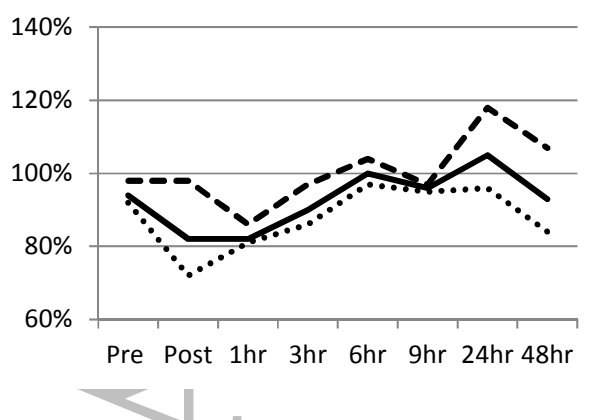

B: $\mathbf{C F X} \mathbf{X}^{\S}$

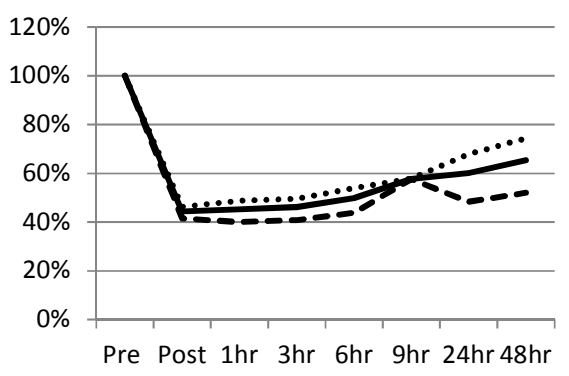

Formatted: Font: (Default) +Body (Calibri)

$11 \mathrm{pt}$

Formatted: Font: Bold

Formatted: Font: Bold

All patients

- - With plasma replacement

..... Without plasma replacement

1

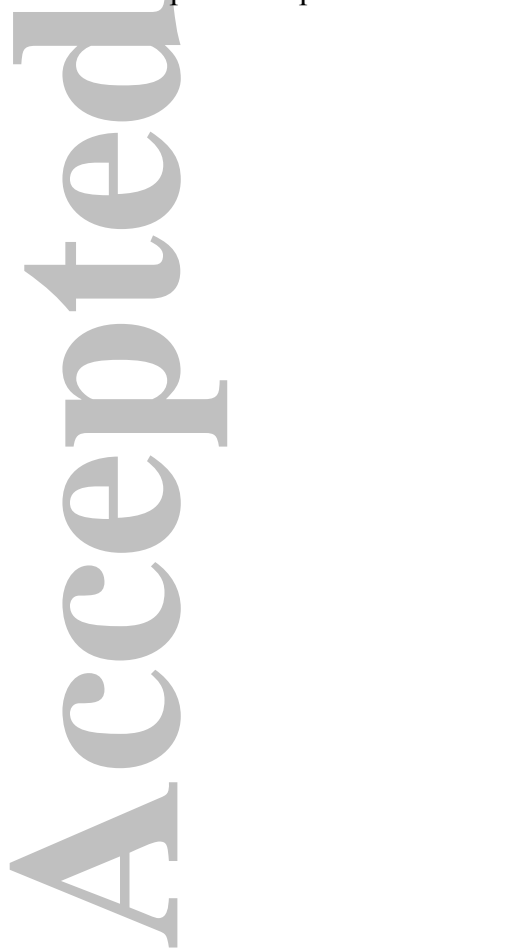




\section{(}

\section{Supplement Figure Legends}

Supplement Figure 1: $\mathrm{nFib}^{\dagger} / \mathrm{FibAg}^{*}$ (normalized fibrinogen activity test result/fibrinogen antigen test result) ratio, $\mathrm{nFib}, \mathrm{INR}$ and $\mathrm{iCa}$ (ionized calcium) levels at Post-TPE (therapeutic plasma exchange).

Supplement Figure 21: The mean level change (\%) based on Pre-TPE*: Therapeutic plasma exchange-level at each time point on patients without anticoagulants. A) $\mathrm{nFib}^{\dagger} / \mathrm{FibAg}^{\star}$ (normalized fibrinogen activity test result/fibrinogen antigen test result)-ratio. B) $\mathrm{CFX}^{\S}$ (coagulation factor $\mathrm{X}$ chromogenic activity) test result. The data is shown in 3 groups, all

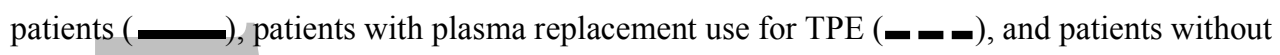

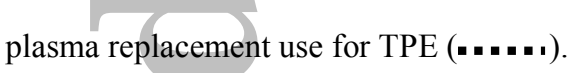

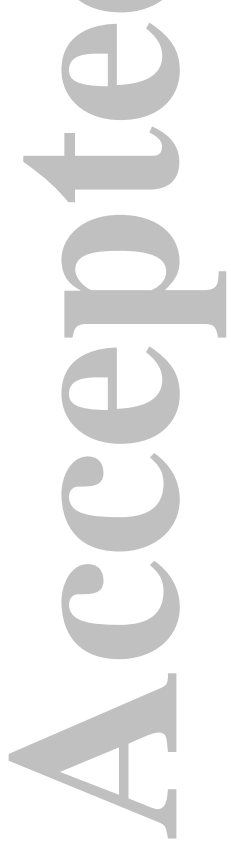

